\title{
Discovering the Core Semantics of Event from Social Media
}

\author{
Weidong Liu ${ }^{\mathrm{a}}$, Xiangfeng Luo ${ }^{\mathrm{a}, *}$, Zhiguo Gong ${ }^{\mathrm{b}}$, Junyu Xuan ${ }^{\mathrm{a}}$, Ngai Meng \\ $\mathrm{Kou}^{\mathrm{b}}$, Zheng $\mathrm{Xu} \mathrm{c}^{\mathrm{c}}$ \\ ${ }^{a}$ Shanghai University, Shanghai, China \\ ${ }^{b}$ University of Macau, Macau, China \\ ${ }^{c}$ The Third Research Institute of Ministry of Public Security, Shanghai, China
}

\begin{abstract}
As social media is opening up such as Twitter and Sina Weibo ${ }^{1}$, large volumes of short texts are flooding on the Web. The ocean of short texts dilutes the limited core semantics of event in cyberspace by redundancy, noises and irrelevant content on the web, which make it difficult to discover the core semantics of event. The major challenges include how to efficiently learn the semantic association distribution by small-scale association relations and how to maximize the coverage of the semantic association distribution by the minimum number of redundancy-free short texts. To solve the above issues, we explore a Markov random field based method for discovering the core semantics of event. This method makes semantics collaborative computation for learning association relation distribution and makes information gradient computation for discovering $k$ redundancy-free texts as the core semantics of event. We evaluate our method by comparing with two state-of-the-art methods on the TAC dataset and the microblog dataset. The results show our method outperforms other methods in extracting core semantics accurately and efficiently. The proposed method can be applied to short text automatic generation, event discovery and summarization for big data analysis.
\end{abstract}

\footnotetext{
* Corresponding author.

Email addresses: liuwd@shu.edu.cn (Weidong Liu), luoxf@shu.edu.cn (Xiangfeng Luo), fstzgg@umac.mo (Zhiguo Gong), xuanjunyu@shu.edu.cn (Junyu Xuan), yb27406@umac.mo (Ngai Meng Kou), xuzheng@shu.edu.cn (Zheng Xu)

${ }^{1}$ Chinese microbloging website http://weibo.com/

Preprint submitted to Journal of ${ }^{A} T_{E} X$ Templates

November 13, 2015
}

(C) 2015. This manuscript version is made available under the Elsevier user license http://www.elsevier.com/open-access/userlicense/1.0/ 
Keywords: core semantics, semantic link network, information gradient

\section{Introduction}

With booming social media, the data explosion of microblog on blogosphere accompanies with hot events. For example, a large volume of microblogs discussed about "USA Boston Marathon bombing", "the US surveillance program 5 PRISM" and so on. Given the microblogs/short texts ${ }^{2}$ about a concrete event, information about the event is unevenly distributed on these "event messages" since some ones might contain much more important and diverse information (e.g., different event time, locations, participants, processes, and opinions) than others (e.g., redundancy and noises in short texts). Besides, these short texts are globally semantic redundant and locally semantic sparse since many short texts contain the same content and local ones only focus on one aspect of the whole event. Understanding the event concisely and thoroughly is impossible when these redundant short texts may crowd out other ones which contain important and diverse information. For example, when we query by keyword "Ebola", Sina Weibo returns redundant Chinese microblogs about "Ebora of salmon" and Twitter returns redundant tweets about "A Italian doctor catches Ebola" as shown in Fig.1, which crowd out many important microblogs which discuss about outbreak, spreading and control of event "Ebola".

Herein, how to automatically discover the core semantics of event from big social media data is a challenging problem, since it is time-consuming and unpractical to manually find out the core semantics of event from big media data.

Existing methods to solve this problem are summarized as follows:

1) Feature-based methods. These methods directly use basic statistic technique on features including word frequency, title words, cure words which are con-

\footnotetext{
${ }^{2}$ There is a word limitation of microblog. For example, each tweet in twitter can't exceed
} 140 words. 


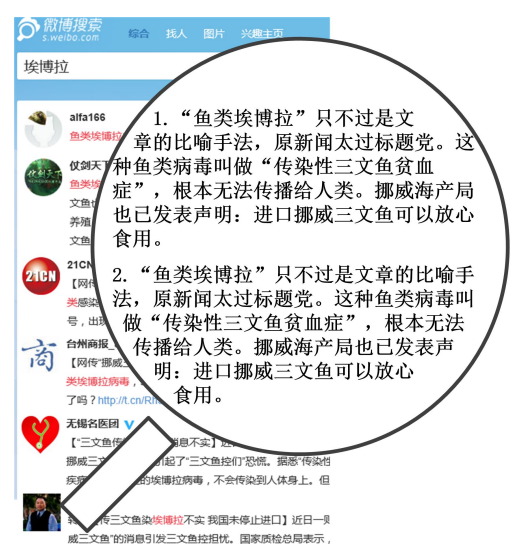

(a) results returned by Sina Weibo

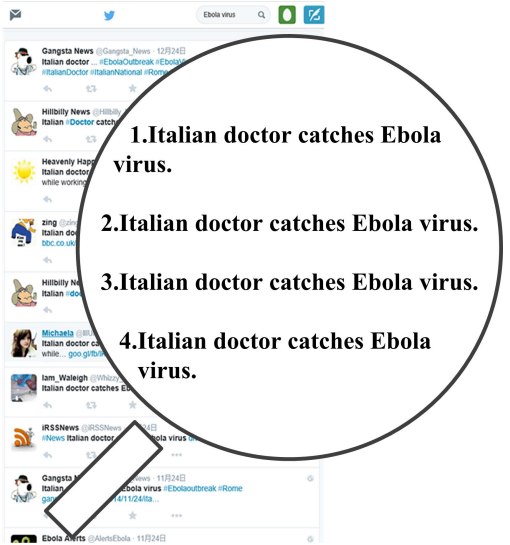

(b) results returned by Twitter

Figure 1: Redundant sentences returned by microblogging services when inputting keyword query "Ebola"

discourse are used to identify core semantics by rhetorical structure analysis, pragmatic analysis, lexical chain, latent semantic analysis[3]. Besides, more features are used in some specified semantics discovery methods whose features include hashtags, timestamps and emotion labels[4].

2) graph-based methods. These methods construct graph where short texts as nodes and text-pairwise relations as edges $[5,6,7,8]$. Top $k$ short texts are selected as core semantics by ranking values of graph-based features or values of Markov random walk on the graph $[5,6,7,8]$. Besides, such methods can be extended into conditional random fields which identify core semantics by labeling sentences, where the sentence label influences the labels of nearby sentences $[9]$.

3) clustering-based methods. These methods cluster short texts into different clusters, and then select some short texts from each cluster to represent the semantics of the cluster[10,11, 12]. The clustering methods include hierarchical clustering, partitional clustering and semantic-based clustering[13]. Besides, some priori knowledge or constraint conditions in specified domain are considered in clustering[14, 15].

4) semantic link-based method. Semantic link-based methods have strong abil- 
ities in semantics organization, semantic community discovery and emerging semantics learning/reasoning[16]. Such methods have been used in semantic representation[17], semantic organization[18], semantic interaction [19, 20], semantic community discovery[21, 22] and semantic linking space for CyberPhysical Society[23, 20].

5) other methods. These methods include Bayesian topic model-based methods[24], Neural Networks-based methods, Decision tree-based methods and so on[25, $26]$.

However, these methods have the following limitations:

1) semantic association loss. The graph-based and cluster-based methods often use vector space model to represent short texts and use vector-based similarity methods. Obviously, these similarity-based methods lost many semantic association relations;

2) high computational cost. The time complexity of most the above methods $[5,6,7,8,10,11,12]$, which have to compute text-pairwise similarity, is $O\left(n^{2}\right)$. It is unpractical when the text number is large in big data;

$\left.{ }_{60} 3\right)$ redundancy-prone results. The above methods pay less attention on the issue of redundancy and result in redundant results since these methods assign almost the same values to alike short texts.

To solve the above limitations, we propose a Markov random field based method for discovering the core semantics of event:

65 1) To avoid semantic association loss, our method makes semantic collaborative computation to learn the whole association relation distribution of an event by small-scale association relations .

2) To reduce computation cost, our method makes probabilistic inference in a limited keyword association link network, rather than text-pairwise computation.

3) To be free of redundancy, our method proposes information gradient computation by maximizing information gradient of $k$ short texts since information gradient decreases when redundancy increases. 
Compared with existing methods, the contributions of our method are summarized as follows:

1) Our method learns association relation distribution by semantic collaborative computation.

2) Our method is efficient by probabilistic inference on semantic association link network.

so 3) Our method obtains redundancy-free core semantics by information gradient computation.

The remainder of the paper is organized as follows. In Section 2, we introduce the preliminaries including some basic definitions and problem formal definition. In Section 3, we propose a framework of Markov random field based method for discovering the core semantics of event. We construct a Markov random field by semantic association collaborative computation, which learns association relation distribution by low-degree relations in Section 4. We propose information gradient computation to maximize coverage of association distribution by the minimum number of redundant-free short texts in Section 5. Experimental re-

90 sults are presented in Section 6. We gives the conclusion and future work in Section 7 .

\section{Preliminary knowledge and problem statement}

Before discussing our method, we first introduce some basic concepts which are thoroughly used in this paper and then propose the problem statement of this paper.

\subsection{Preliminary knowledge}

Semantic representation and inference are two major issues for discovering the core semantics of event. We introduce two basic models in semantic representation and probabilistic inferences. 

$[17,27,28,29,30,31,32,33]$, where each concept consists of association relations. Inspired by [17], we adopt association relations to represent an event as follows:

Definition 1 (Event Power Serial Representation, E-PSR). E-PSR is represented as,

$$
\begin{gathered}
E-P S R=\left\{\Phi_{k} \mid 0 \leq k \leq 2\right\} \\
\Phi_{k}=\left\{\phi_{k, i}\right\}
\end{gathered}
$$

where $\Phi_{k}$ denotes a k-degree association relation set; $\phi_{k, i}=w_{0}^{(k, i)}, w_{1}^{(k, i)}, \ldots, w_{k-1}^{(k, i)} \rightarrow$ $w_{k}^{(k, i)}$ is a k-degree association relation with support value which is calculated by,

$$
\sup \left(\phi_{k, i}\right)=\frac{\sum_{s_{l} \in e} I\left(w_{0}^{(k, i)}, w_{1}^{(k, i)}, \ldots, w_{k}^{(k, i)} \mid s_{l}\right)}{\sum_{s_{l} \in e} I\left(s_{l} \mid s_{l}\right)}
$$

where $I(A \mid B)$ is an indicator function whose value is 1 if $A \subseteq B$ and 0 otherwise; $e=\left\{s_{l} \mid 1 \leq l \leq n\right\}$ denotes an event which consists of $n$ sentences.

For example, an event contains two short texts, $e=\left\{s_{1}, s_{2}\right\}$ :

$s_{1}$ : That boy stands on the left, whose t-shirt is red.

$s_{2}$ : Two girls stand on the right, whose skirts are also red.

115 E-PSR of the event $e$ includes association relations as follows:

$\Phi_{0}=\{$ red $\} ; \Phi_{1}=\{$ boy $\rightarrow$ left, girl $\rightarrow$ right, boy $\rightarrow$ t-shirt, girl $\rightarrow$ skirt $\}$.

E-PSR simply obtains low-degree association relation distribution $\left\{\Phi_{k} \mid 0 \leq\right.$ $k \leq 2\}$ rather than the whole association relation distribution $\left\{\Phi_{k} \mid 0 \leq k\right\}$, since how to obtain $\left\{\Phi_{k} \mid k>2\right\}$ is still a unsolved problem. As such, we may ask the following question: Is the above problem solved by inference based on probabilistic model?

Markov random field model(MRF) is an undirected probabilistic graphical model. We propose event Markov random field for semantic representation and inference. 
Definition 2 (Event Markov Random Field, E-MRF). E-MRF is represented by,

$$
E-M R F=<G, P(X)>
$$

where $G=<X, E>$ is an undirected graph, where $X$ denotes a set of random variables and $E$ denotes a set of dependence relations between $X ; P$ denotes a joint probability distribution over $X$, which is calculated by:

$$
P(X)=\mu_{0} \Pi_{c_{i} \in C} \Psi\left(X_{c_{i}}\right)
$$

130 the core semantics of event.

Supposing an event $e=\left\{s_{l} \mid 1 \leq l \leq n\right\}$, consists of $n$ sentences, such as microblogs, tweets or comments, the $k$ sentences discovered from $e$ should satisfy the following properties:

where $I\left(\phi_{k, i} \mid x\right)$ is an indicator function whose value is 1 if $x$ is consistent with $\phi_{k, i}$ and 0 otherwise; $x$ is consistent with $\phi_{k, i}$ if $x_{\left(w_{w_{t}}^{(k, i)}\right)}=1, \quad\left(w_{w_{t}}^{(k, i)} \in\right.$ $\left.\phi_{k, i}, \quad x_{\left(w_{w_{t}}^{(k, i)}\right)} \in x\right)$.

\subsection{Problem statement}

In this paper, our task is to learn association relation distribution of an event and to cover the distribution by $k$ sentences as the core semantics of event. The $k$ sentences which can cover the association relation distribution of an event is 
1) Priority for sentence with frequently discussed content, since such sentences include more core association relations.

2) Priority for sentence with new content, since such sentences provide user with more information about the event.

Definition 3 (information gradient of $\boldsymbol{k}$-sentences, $\left.I G\left(S_{k}\right)\right)$. $I G\left(S_{k}\right)$ re-

flects how frequent and how novel the content of $k$ sentences is in an event, which is calculated by,

$$
I G\left(S_{k}\right)=I G\left(S_{k-1}\right)+I G\left(s_{(k)} \mid S_{k-1}\right)
$$

where $S_{k}=\left\{s_{(l)} \mid 1 \leq l \leq k\right\}$ denotes a set of $k$ sentences; $s_{(l)}$ denotes the $l^{\text {th }}$ sentence; $I G\left(s_{(k)} \mid S_{k-1}\right)$ denotes conditional information gradient of the sentence $s_{(k)}$ conditioned on $S_{k-1}$.

$I G\left(s_{(k)} \mid S_{k-1}\right)$ is approximated 0 when $s_{(k)}$ provides no novel or indifferent content conditioned on $S_{k-1}$.

$$
I G\left(s_{(k)} \mid S_{k-1}\right) \approx 0 \text { if } I G\left(S_{k}\right)-I G\left(S_{k-1}\right) \approx 0
$$

To satisfy above properties, the core semantic of event is defined as,

Definition 4 (Core Semantics of Event, $C S(e)$ ). the core semantic of event is obtained by maximizing information gradient of $k$ sentences,

$$
C S(e)=\arg \max _{\left|S_{k}\right|=k} I G\left(S_{k}\right)
$$

We list the notations of the above definitions in table 1, which are thoroughly used in this paper.

\section{Proposed method}

To solve the problem defined in equation 9, a framework of Markov random field based method for discovering core semantics is shown in Fig.2. In the following, we introduce the whole framework by 4 steps. $1^{\text {st }}$ and $2^{\text {nd }}$ steps mainly obtain short texts for each event. The most adopted algorithms to obtain 
Table 1: Notations used in the paper

\begin{tabular}{|c|l|}
\hline Symbols & Description \\
\hline \hline$\phi_{k, i}$ & $\phi_{k, i}=w_{0}^{(k, i)}, w_{1}^{(k, i)}, \ldots, w_{k-1}^{(k, i)} \rightarrow w_{k}^{(k, i)}$ is a k-degree association relation \\
\hline$\Phi_{k}$ & $\Phi_{k}=\left\{\phi_{k, i}|i<| \Phi_{k} \mid\right\}$ denotes a k-degree association relation set \\
\hline$E-P S R$ & $E-P S R=\left\{\Phi_{k} \mid 0 \leq k \leq 2\right\}$ is a set of association relations set \\
\hline$X$ & $X=\left\{X_{w_{i}}|0 \leq i \leq| X \mid\right\}$ denotes a random variable set \\
\hline$G=<X, E>$ & $G=<X, E>$ is an undirected graph, where $E$ denotes dependence of $X$ \\
\hline$c_{i}$ & $c_{i}$ is a maximal clique which is a full connective sub-graph of $G$ \\
\hline$C$ & $C=c_{i}$ is a set of maximal cliques \\
\hline$X_{c_{i}}$ & a maximal clique random variable of $c_{i}$ \\
\hline$\Psi\left(X_{c_{i}}\right)$ & a non-negative potential function over $X_{c_{i}}$ \\
\hline$\mu_{i}$ & a parameters of Markov random field \\
\hline$P(X)$ & joint probability of $X$ \\
\hline$\widehat{X}$ & $\widehat{X}$ is a sub-set of variables $X$ \\
\hline$P(\widehat{X})$ & marginal probability of $\widehat{X}$ compared with joint probability $P(X)$ \\
\hline$E-M R F$ & $E$ - $M R F=<G, P(X)>$ denotes an event Markov random model \\
\hline$I G\left(S_{k}\right)$ & information gradient of $S_{k}=\left\{s_{(l)} \mid 1 \leq l<k\right\}$ \\
\hline$I G\left(s_{(k)} \mid S_{k-1}\right)$ & conditional information gradient of a sentence $s_{(k)}$ conditioned on $S_{k-1}$ \\
\hline \hline
\end{tabular}

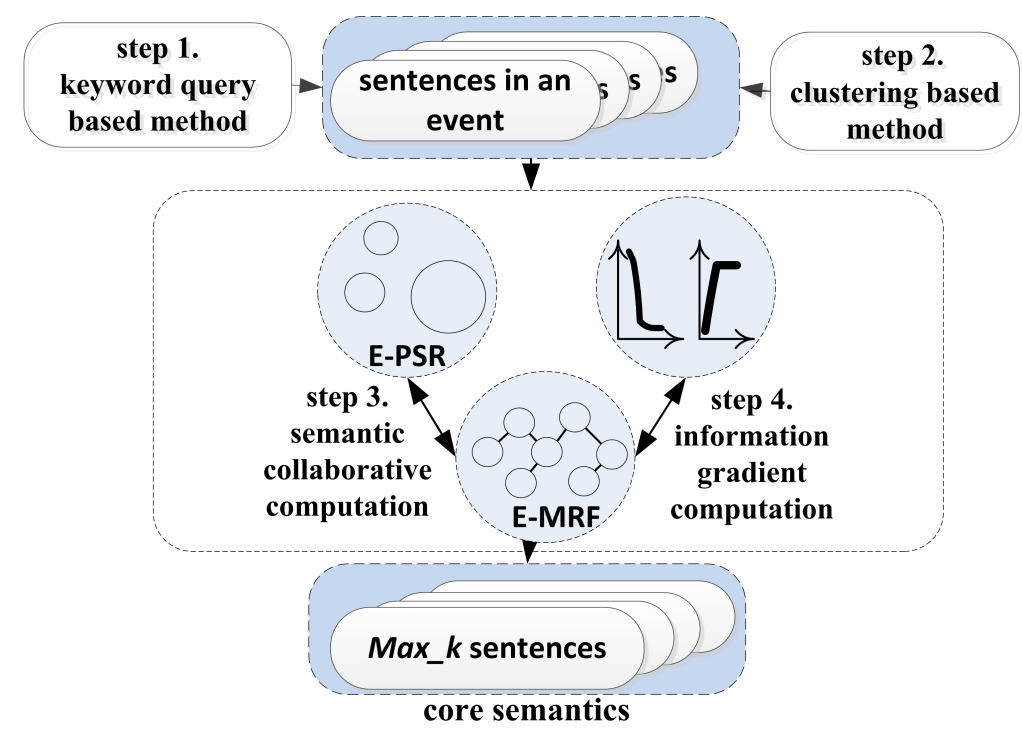

Figure 2: A framework of Markov random field based method for discovering the core semantics of event 
these short texts are: 1) query based algorithms, users query some keywords to get related sentences of an event; 2) event detection or clustering based algorithms, such algorithms discover sentences which belong to different events ${ }_{175}[34,35,36]$. To ensure the data is valid, we apply the state-of-the-art event discovery methods to reduce the possible negative effects on core semantics discovery $[37,38,39,40]$. Besides, association relation based representation in our model can further reduces these adverse effects caused by noise and irrelevant short texts. $1^{\text {st }}$ and $2^{\text {nd }}$ steps are not the focus of this paper.

We mainly focus on $3^{\text {th }}-4^{\text {th }}$ steps for discovering core semantics of an event.

Obtaining semantic association relation distribution is a basic issue for core semantics discovery. Our Markov random field based method collaborates with power serial representation model to learn the association relation distribution of an event. $3^{\text {th }}$ step gives semantic collaborative computation between E-MRF and E-PSR, which mutually benefits each other: E-PSR reduces computational cost of construction of E-MRF by low-degree association relations $\left\{\Phi_{k} \mid 0 \leq k \leq\right.$ $2\}$; E-MRF extends E-PSR with inference ability on high-degree association relation $\left\{\Phi_{k} \mid k>2\right\}$.

Although, the above method has built a probabilistic graphic model which has strong abilities in semantic representation and inference, it assigns alike sentences similar probability values no matter how redundant they are. Obviously, such method is redundancy-prone.

To obtain redundancy-free results, $4^{\text {th }}$ step makes information gradient computation, which assigns redundant sentences with lower conditional information 195 gradient by equation 8 since these redundant sentences contain little novel content. If $k$ sentences can maximize information gradient defined in equation 9 , the $k$ sentences are the core semantics of event which maximally covers the association relation distribution. 


\section{Semantic collaborative computation}

200 laborates E-MRF with E-PSR because: 1) semantic association consistence. EMRF keeps association consistence with E-PSR by association relations which cover almost 90\% semantic association [17]; 2) learning efficiency improvement. E-PSR reduces the graphic scale of E-MRF with smaller and sparser associaE-MRF

\subsection{Collaborative computation of E-MRF with E-PSR}

E-MRF is an undirected probabilistic graphic model and E-PSR is an association relation set. Structural consistence and association consistence between tion. To guarantee these consistences, we collaborate E-MRF with E-PSR by structural collaborative computation and potential value collaborative computation.

\subsubsection{Structural collaborative computation}

Structural collaborative computation mainly solves issues: 1) how dose EPSR form graphic structure of E-MRF; 2) what's the parameter structure of E-MRF formed by E-PSR. Referred to a factor graph theory [41], given $\mathrm{E}-\mathrm{PSR}=\left\{\Phi_{k} \mid 0 \leq k \leq 2\right\}$, we solve the above issues by $1^{\text {st }}-4^{\text {th }}$ steps. For 1 ), we form an undirected graph structure by $1^{\text {st }}-2^{\text {nd }}$ steps; For 2 ), we form the parameter structure by $3^{\text {th }}-4^{\text {th }}$ steps.

step 1. add a variable $X_{w_{i}}$ in E-MRF, only if keywords $w_{i}$ currents in E-PSR;

step 2. link $X_{w_{i}}$ and $X_{w_{j}}$ in E-MRF, only if $w_{i}$ and $w_{j}$ co-occur in an association relations in E-PSR;

step 3. map each association relation into a maximal clique, only if the keywords in association relations are contained by the maximal clique; 
step 4. set the parameter structure for each maximal clique variable by,

$$
\Psi_{X_{c_{i}}}=\Pi_{\phi_{i} \in\left\{\Phi_{k} \mid 0 \leq k \leq 2\right\}} \mu_{i}^{I\left(X_{c_{i}} \mid \phi_{i}\right)}
$$

where $X_{c_{i}}$ denotes a maximal clique variable; $\mu_{i}$ is a parameter of an association relation $\phi_{i}$ in E-PSR; $I\left(X_{c_{i}} \mid \phi_{i}\right)$ is an indicator function whose outcome is 1 when association relation $\phi_{i}$ is consistent with the maximal clique variable $X_{c_{i}}$, 0 otherwise.

According to $1^{s t}-4^{t h}$ steps, we propose a structural collaborative algorithm 1.

Algorithm 1 structural collaborative algorithm

Input: an event, $e=\left\{s_{l} \mid 1 \leq l<n\right\}$

Output: a graphic structure $G=<X, E>$ and a parameter structure $\left\{\Psi_{X_{c_{i}}}\right\}$ of E-MRF

1. mine E-PSR by algorithm [17]

2. form $G=<X, E>$ by $1^{\text {st }}-2^{\text {nd }}$ steps

3. form $\left\{\Psi_{X_{c_{i}}}\right\}$ by $3^{\text {th }}-4^{\text {th }}$ steps

4. return $G=<X, E>$ and $\left\{\Psi_{X_{c_{i}}}\right\}$

\subsubsection{Potential value collaborative computation}

To guarantee semantic association consistence between E-PSR and E-MRF, ues of E-MRF from E-PSR. It is easily found that $\sup \left(\phi_{k, i}\right)$ defined in equation 3 is unbiased estimation of marginal probability $P\left(\phi_{k, i}\right)$ defined in equation 6 by,

$$
\left|\Sigma_{x} P(x) I\left(\phi_{k, i} \mid x\right)-\sup \left(\phi_{k, i}\right)\right|<\varepsilon
$$

where $\sup \left(\phi_{k, i}\right)$ denotes the support value of $\phi_{k, i}$.

The parameter $\mu_{i}$ defined in equation 10 should satisfies equation 11 . Using local item sets to construct a MRF model is first proposed by Pavlov [42], 
we adopted an iterative scaling (IS) algorithm [43, 44, 45] to learn parameters $\left\{\mu_{i}|0 \leq i \leq| E-P S R \mid\right\}$ by,

$$
\begin{gathered}
\mu_{0}^{t+1}=\mu_{0}^{t} \times \frac{1-\sup \left(\phi_{i}\right)}{1-P^{t}\left(\phi_{i}\right)} \\
\mu_{i}^{t+1}=\mu_{i}^{t} \times \frac{\sup \left(\phi_{i}\right) \times\left(1-P^{t}\left(\phi_{i}\right)\right)}{P^{t}\left(\phi_{i}\right) \times\left(1-\sup \left(\phi_{i}\right)\right)} \quad(0<i)
\end{gathered}
$$

Herein, we propose a potential value collaborative algorithm 2 .

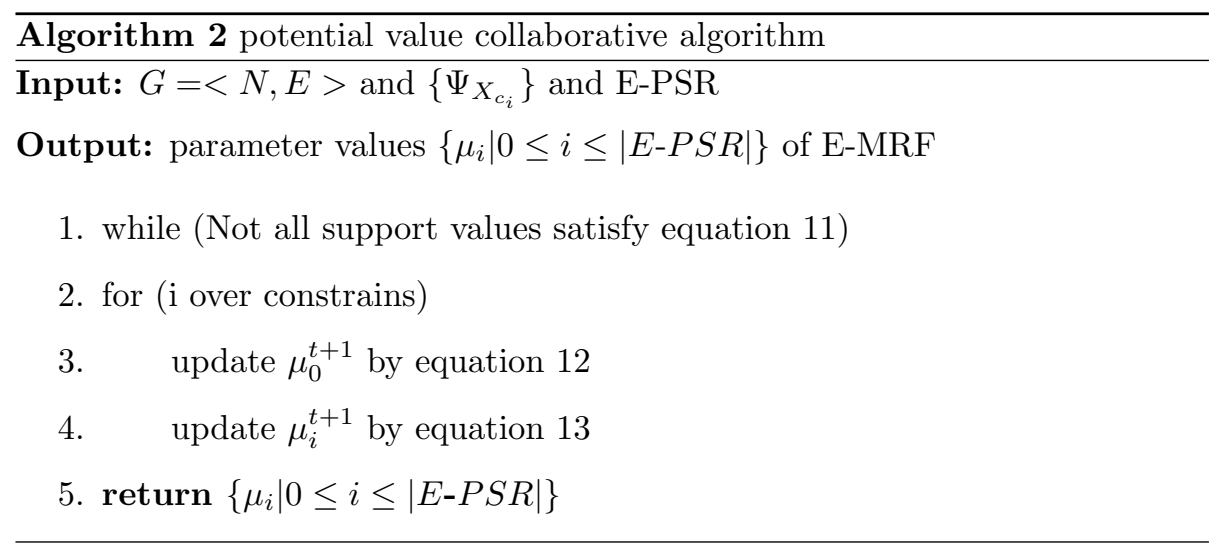

\subsection{Basic semantic computation in $E-M R F$}

Given $E-M R F=<G, P(X)>$ learned by algorithm 1-2, we had obtain the joint probability distribution of $X$ since we calculate probability of sub-variables $\widehat{X}$ by,

$$
P(\widehat{X})=\Sigma_{x} P(x) I(\widehat{X} \mid x)
$$

250

where $\widehat{X}$ is a subset of random variables $x ; I(\widehat{X} \mid x)$ is an indicator function with outcome 1 when $\widehat{X}$ is consistent with $x, 0$ otherwise.

Based on equation 14, we make some basic semantic computations. For example, we can calculate how frequently a sentence is discussed by,

$$
P\left(s_{i}\right)=\Sigma_{x} P(x) I\left(s_{i} \mid x\right)
$$

where $I\left(s_{i} \mid x\right)$ is an indicator function with outcome 1 when $s_{i}$ is consistent with $255 \quad x, 0$ otherwise. 
A common issue among equations $11-15$ is to calculate the marginal probability of E-MRF. Junction tree algorithm is a general probabilistic inference framework for calculating joint probability, marginal probability and condition probability [46] by decomposing a global joint probability computation into a linked set of local computations. Referring to [46], we adopt the junction tree algorithm to calculate marginal probability.

If a sentence with higher frequency is selected as core semantics by equation 15 , large number of alike sentences will be selected as well since their similar contents and thus result in undesirable redundancy.

\section{Information gradient of k-sentences}

Redundancy conflicts with cognitive psychology [47], since undue redundancy has limited effect on memory activity. For human beings, repeated memorizing a word or sentence content has limited effect in human memory process as shown in Fig.3. Fig.3(a) shows that memory activity value changes with repetition. The memory activity significantly increases with increasing repetition before about 23 times and then gets stable thereafter. Fig.3(b) shows that gradient of the memory activity changes with increasing repetition. The memory gradient gradually decreases into 0 when the repetition increases.

\subsection{The computation of information gradient}

Inspired by memory activity lines in Fig.3, we propose information gradient which decreases as redundancy increase as follows.

Definition 5 (Information Gradient of Association Relation, $I G_{t}\left(\phi_{i}\right)$ ). $I G_{t}\left(\phi_{i}\right)$ reflects the amount of association information in $\phi_{i}$ when it is described at $t^{\text {th }}$ time

$$
I G_{t}\left(\phi_{i}\right)= \begin{cases}1-\operatorname{EXP}\left(-\lambda_{i} \times t^{\alpha}\right), & t=1 ; \\ \operatorname{EXP}\left(\lambda_{i} \times\left(t^{\alpha}-(t-1)^{\alpha}\right)\right), & t>1 .\end{cases}
$$

280

$$
t=\Sigma_{s_{(l)} \in S_{k-1}} I\left(\phi_{i} \mid s_{(l)}\right)
$$



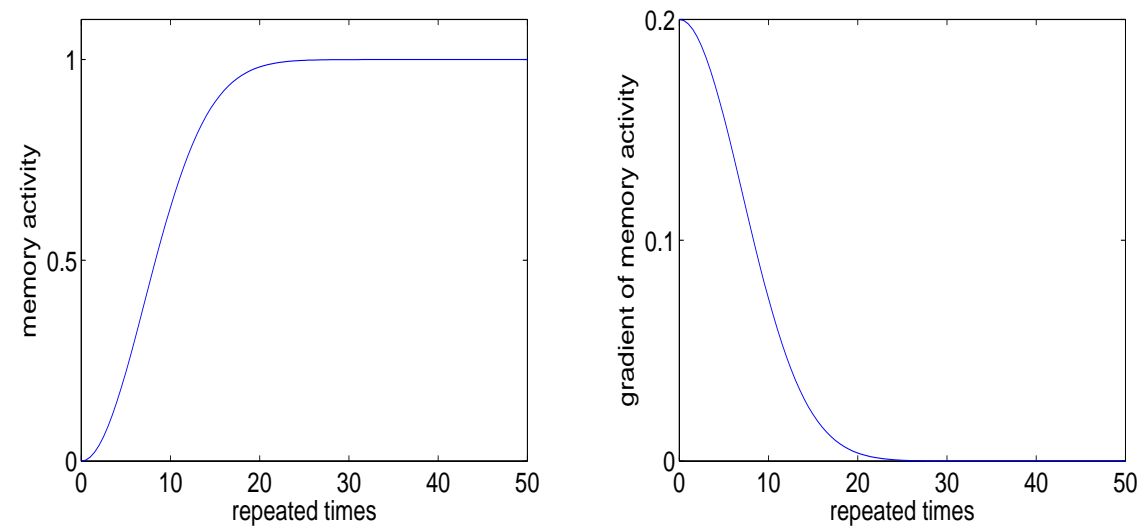

(a) memory activity changes with repeated (b) memory gradient changes with repeated times times

Figure 3: memory activity trend line changes with repetition

where $I G_{t}\left(\phi_{i}\right) \rightarrow[0,1] ; I\left(\phi_{i} \mid s_{(l)}\right)$ is an indicator function, whose outcome is 1 when $\phi_{i}$ is consistent with $s_{(l)} ; t$ is the repetition of $\phi_{i}$ described by $S_{k-1}=$ $\left\{s_{(l)} \mid 1 \leq l<k-1\right\} ; \lambda_{i}$ is a decay rate of $\phi_{i}$.

From equation 16-17, we know that $I G_{t}\left(\phi_{i}\right)$ value is conditioned on repetition $t$ of $\phi_{i}$, which has the following properties by following lemmas:

Lemma 6. $I G_{t}\left(\phi_{i}\right)$ gradually decreases with $t$ increases.

$$
I G_{t}\left(\phi_{i}\right)>I G_{t+1}\left(\phi_{i}\right)
$$

Proof. Suppose $\sigma=I G_{t}\left(\phi_{i}\right)-I G_{t+1}\left(\phi_{i}\right)$ and $\rho(t)=E X P\left(-\lambda \times t^{\alpha}\right)$

Then,

$\sigma=2 \times E X P\left(-\lambda \times t^{\alpha}\right)-E X P\left(-\lambda \times(t-1)^{\alpha}\right)-E X P\left(-\lambda \times(t+1)^{\alpha}\right)$

$290 \quad \sigma=2 \times \rho(t)-\rho(t-1)-\rho(t+1)$

since $\rho(x)=\operatorname{EXP}\left(-\lambda \times t^{\alpha}\right)$ is a concave function, $\sigma<0$ and therefore lemma 6 is proofed.

Lemma 7. $I G_{t}\left(\phi_{i}\right)$ decreases more sharply with $t$ increases.

Suppose $F(x)=\Sigma_{x} I G_{x}\left(\phi_{i}\right)=1-E X P\left(-\lambda \times x^{\alpha}\right)$ and $t_{2}>t_{1}$

$\left(F\left(t_{2}+h\right)-F\left(t_{2}\right)\right) /\left(h \times\left(F(\propto)-F\left(t_{2}\right)\right)\right)>\left(F\left(t_{1}+h\right)-F\left(t_{1}\right)\right) /\left(h \times\left(F(\propto)-F\left(t_{1}\right)\right)\right)$ 
where $\Psi_{S_{k-1}}\left(X_{c_{i}}\right)$ is refereed by equation 20

\subsection{The maximization of information gradient}

Referred as equation 22 and equation $9, I G\left(s_{(k)} \mid S_{k-1}\right)$ is calculated by,

$$
I G\left(s_{(k)} \mid S_{k-1}\right)=\Sigma_{x} P_{S_{k-1}}(x) I\left(s_{(k)} \mid x\right)
$$

where $P_{S_{k-1}}(x)$ denotes a joint probability distribution of $x$ before adding $s_{(k)}$; $s_{(k)}, 0$ otherwise.

Compared equation 20 with equation 10, it is found that $I G_{t}\left(\phi_{i}\right)$ exerts influence on parameters $\mu_{i}$ in equation 20 .

Supposing $S_{k-1}=\left\{s_{l} \mid 1 \leq l \leq k-1\right\}$ has described the association relation distribution before adding $s_{(k)}$, the association relation distribution is calculated by,

$$
P_{S_{k-1}}(X)=\mu_{0} \Pi_{c_{i} \in C} \Psi_{S_{k-1}}\left(X_{c_{i}}\right)
$$

$I\left(s_{(k)} \mid x\right)$ is an indicator function, whose outcome is 1 when $x$ is consistent with 
Lemma 8. $I G\left(s_{(i)} \mid S^{\prime \prime}\right) \geq I G\left(s_{(i)} \mid S^{\prime}\right)$ if $S^{\prime \prime} \subseteq S^{\prime}$

Proof. $I G\left(s_{(k)} \mid S^{\prime}\right)=P_{S^{\prime}}(X) I\left(s_{(k)} \mid x\right)$

$I G\left(s_{(k)} \mid S^{\prime \prime}\right)=P_{S^{\prime \prime}}(X) I\left(s_{(k)} \mid x\right)$

320 According lemma $6, I G_{t^{s^{\prime \prime}}}\left(\phi_{i}\right) \geq I G_{t^{s^{\prime}}}\left(\phi_{i}\right)$, since $t^{s^{\prime \prime}}<t^{s^{\prime}}$

so $I G\left(s_{(i)} \mid S^{\prime \prime}\right) \geq I G\left(s_{(i)} \mid S^{\prime}\right)$, lemma 8 is proofed.

Referred as equation 9 in problem definition, the core semantics of event is obtained by maximizing information gradient of $k$ sentences is calculated by,

$$
C S(e)=\arg \max _{\left|S_{k}\right|=k} I G\left(S_{k}\right)
$$

where $G\left(S_{k}\right)=I G\left(S_{k-1}\right)+I G\left(s_{(k)} \mid S_{k-1}\right) ; I G\left(s_{(k)} \mid S_{k-1}\right)=\Sigma_{x} P_{S_{k-1}}(x) I\left(s_{(k)} \mid x\right)$

If $I G\left(S_{k}\right)$ satisfies lemma 8 , then $I G(S)$ is a submodular function [48]. For a submodular function, it has been proofed that the CELF method can obtain a near-optimal solution for maximizing information gradient of $k$ sentences [48]. The equation 24 is maximized by the algorithm 3 .

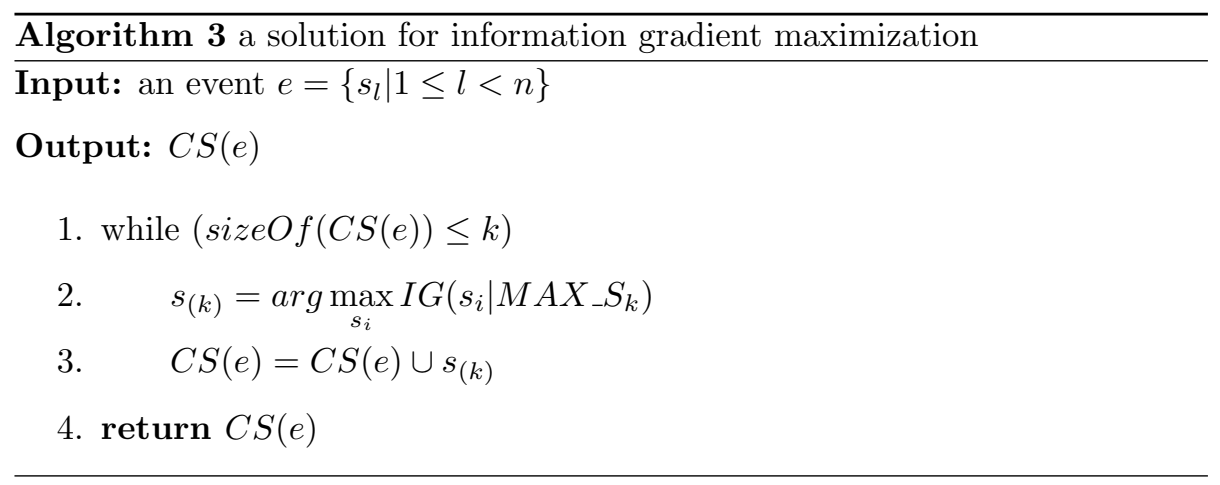

\section{Experiments}

In this section, we conduct some experiments to validate the correctness of our method. 


\subsection{Datasets and evaluation measurement}

To evaluate our method, we use TAC2008-TAC2010 3 as dataset 1 and realworld microblog data crawled from Sina Weibo as dataset 2. Table 2 gives some statistics about the dataset 1 and dataset 2 .

1) Dataset 1 includes 138 topics where each topic has a topic statement (title and narrative) and 20 relevant documents which have been divided into 2 sets: document set $\mathrm{A}$ and document set $\mathrm{B}$. In this paper, we use set $\mathrm{A}$ in our experiments, where each topic has average 262 sentences and 4 manually generated summaries with 100-word length respectively.

2) Dataset 2 includes 20 events with total 725300 microblogs. For each event, we crawl microblogs in 30 days since its beginning timestamp. Besides we also collect the titles of news about these events from Baidu news ${ }^{4}$ in the same period. More details are shown in Table 3

Table 2: Description of datasets

\begin{tabular}{|c|c|c|}
\hline \multirow{2}{*}{ Dataset source } & Dataset 1 & Dataset 2 \\
\cline { 2 - 3 } & TAC2008-TAC2010 & microblogs \\
\hline \hline Total \# topics/event & 138 & 20 \\
\hline Avg.\# sentences in a topic & 262 & 36255 \\
\hline Avg.\# keywords & 840 & 21367 \\
\hline Total \# sentences & 128414 & 725300 \\
\hline \hline
\end{tabular}

\section{Baseline methods}

We compare our methods with following state-of-the-art methods:

1) Cluster-based Conditional Markov random Walk Model (ClusterCMRW) [7]: it clusters sentences first and then ranks sentences in each clustering.

2) Cluster-based HITS Model (ClusterHITS) [7]: it clusters sentences first and then regards each clustering as hub and each sentence as authority. It uses hub value to rank clusters and use the authority value to rank sentences.

\footnotetext{
${ }^{3}$ http://www.nist.gov/tac/data/index.html

${ }^{4}$ http://news.baidu.com
} 
Table 3: Description of microblog dataset in Sina Weibo

\begin{tabular}{|c|c|c|c|}
\hline Event & $\begin{array}{l}\text { Beginning } \\
\text { Timestamp }\end{array}$ & \#microblogs & $\begin{array}{l}\text { \#Title of } \\
\text { Baidu News }\end{array}$ \\
\hline The crisis in the Korean Peninsula & 2013-03-08 & 105150 & 7288 \\
\hline USA Boston Marathon bombing & 2013-04-15 & 42987 & 4531 \\
\hline $\begin{array}{l}\text { the US surveillance program } \\
\text { PRISM }\end{array}$ & 2013-08-22 & 31939 & 2912 \\
\hline The crisis in Syria & 2013-09-05 & 84172 & 9875 \\
\hline $\begin{array}{l}\text { China's declaration of an air de- } \\
\text { fense zone }\end{array}$ & $2013-11-23$ & 40414 & 3280 \\
\hline $\begin{array}{l}\text { China's first moon rover, Yutu, or } \\
\text { Jade Rabbit }\end{array}$ & 2013-12-02 & 20621 & 1078 \\
\hline The crisis in Ukraine & $2014-02-22$ & 13317 & 3149 \\
\hline $\begin{array}{l}\text { Malaysia Airline's flight } 370 \text { disap- } \\
\text { peared }\end{array}$ & 2014-03-08 & 62126 & 10368 \\
\hline $\begin{array}{l}\text { Declaration of independence of Au- } \\
\text { tonomous Republic of Crimea }\end{array}$ & 2014-03-16 & 72079 & 5314 \\
\hline $\begin{array}{l}\text { Sunflower Student Movement in } \\
\text { Taiwan }\end{array}$ & 2014-03-18 & 2358 & 153 \\
\hline South Korea's ferry accident & 2014-04-16 & 34388 & 2172 \\
\hline 981 drilling platform & 2014-05-27 & 2552 & 257 \\
\hline $\begin{array}{l}\text { Establishment of Shanghai Pilot } \\
\text { Free Trade Zone }\end{array}$ & 2013-09-30 & 1562 & 819 \\
\hline 2014 FIFA World Cup Brazil & 2014-06-13 & 128472 & 22206 \\
\hline Islamic State in Iraq and Syria & 2014-06-29 & 4612 & 2037 \\
\hline $\begin{array}{l}\text { The crash of Malaysia Airline's } \\
\text { Boeing- } 777\end{array}$ & $2014-07-17$ & 16248 & 2591 \\
\hline Scottish referendum & 2014-08-05 & 1681 & 234 \\
\hline $\begin{array}{l}\text { WHO issued that Ebola became } \\
\text { an international public health emer- } \\
\text { gency }\end{array}$ & 2014-08-08 & 7533 & 4332 \\
\hline Taiwan gutter oil scandal & 2014-09-04 & 12692 & 1457 \\
\hline $\begin{array}{l}\text { Alibaba will begin I.P.O. Process in } \\
\text { U.S. }\end{array}$ & 2014-09-09 & 40399 & 4721 \\
\hline $\begin{array}{l}\text { North Korean government an- } \\
\text { nounced the withdrawal of nonag- } \\
\text { gression treaty with South Korea }\end{array}$ & 2013-03-08 & 105150 & 7288 \\
\hline
\end{tabular}




\section{Evaluate measurement}

We use a widely used evaluation toolkit ROUGE [49] for evaluation. It measures summaries by counting the overlaps between the system generated summaries and human-written summaries as reference summaries. We mainly use ROUGE1, ROUGE-2 and ROUGE-SU4 in our experiments.

ROUGE-N is calculated as follows:

$$
\text { ROUGE- } n=\frac{\Sigma_{s \in \text { ref }} \Sigma_{n \text {-gram } \in \text { s }} I(n \text {-gram } \mid \text { ref }, \text { gen })}{\Sigma_{s \in \text { ref }} \Sigma_{n \text {-gram } \in \text { s }} I(n \text {-gram } \mid \text { ref })}
$$

Where $n$ denotes word length of n-gram; I(n-gram|ref,gen $)$ is an indicator

function whose values is 1 when $n$-gram in the generated summary and reference summaries; $I$ (n-gram|ref) outcomes 1 when $n$-gram in reference summaries.

ROUGE-SU4 is calculated as follows:

$R O U G E-S U 4=\frac{\Sigma_{s \in \text { ref }}\left(\Sigma_{\text {skip } 2 \text {-gram } \in \text { s }} I(\text { skip2-gram } \mid \text { ref }, \text { gen })+\Sigma_{1 \text {-gram } \in \text { s }} I(1 \text {-gram } \mid \text { ref }, \text { gen })\right)}{\left.\Sigma_{s \in \text { ref }} \Sigma_{\text {skip } 2 \text {-gram } \in \text { s }} I(\text { skip2-gram } \mid \text { ref })+\Sigma_{1 \text {-gram } \in S} I(1-\text { gram } \mid \text { ref })\right)}$

Where S4 denotes skip-bigram of any word pair in sentences whose word distance is at most $4 ; U$ denotes unigram.

\subsection{Experimental setup}

For evaluation of our method, we use dataset 1 and dataset 2 to conduct the experiments. The sentences in dataset 1 and dataset 2 are tokenized and stemmed by Stanford parser tools ${ }^{5}$. Our method for discovering core semantics is conducted as follows:

1) To obtain distribution of association semantics and enable semantics inference by low-degree association relations, we construct E-MRF model by a graph structure algorithm 1 and a potential value collaborative algorithm 2 in section 4 .

3752 ) To solve problem of maximizing information gradient, we select $k$ sentence as the core semantics of event by algorithm 3 in section 5 . 


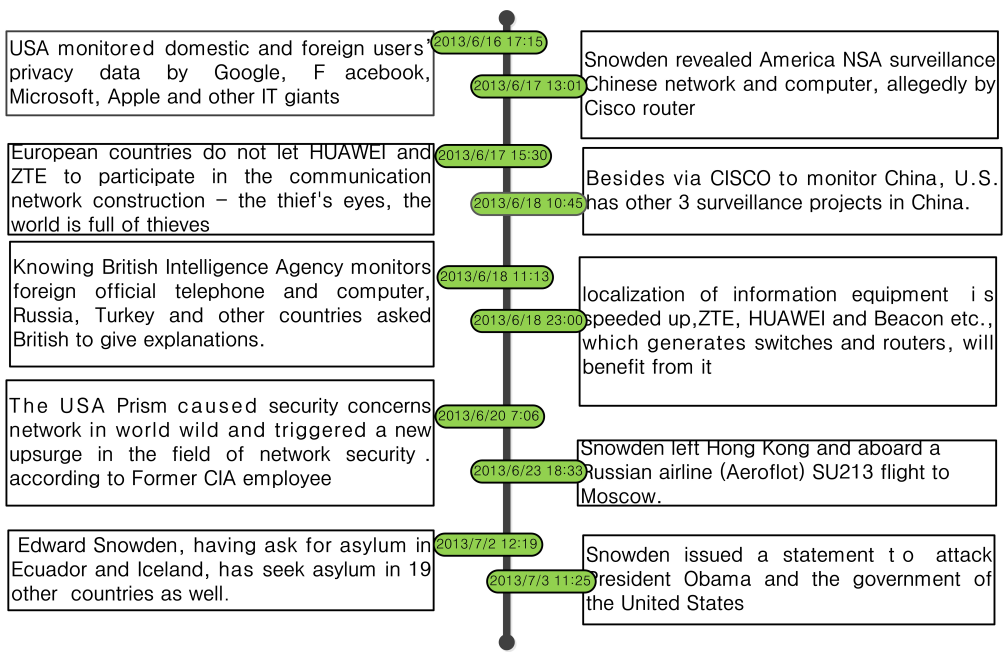

Figure 4: 10 sentences as the core semantics of event "The US surveillance program PRISM"

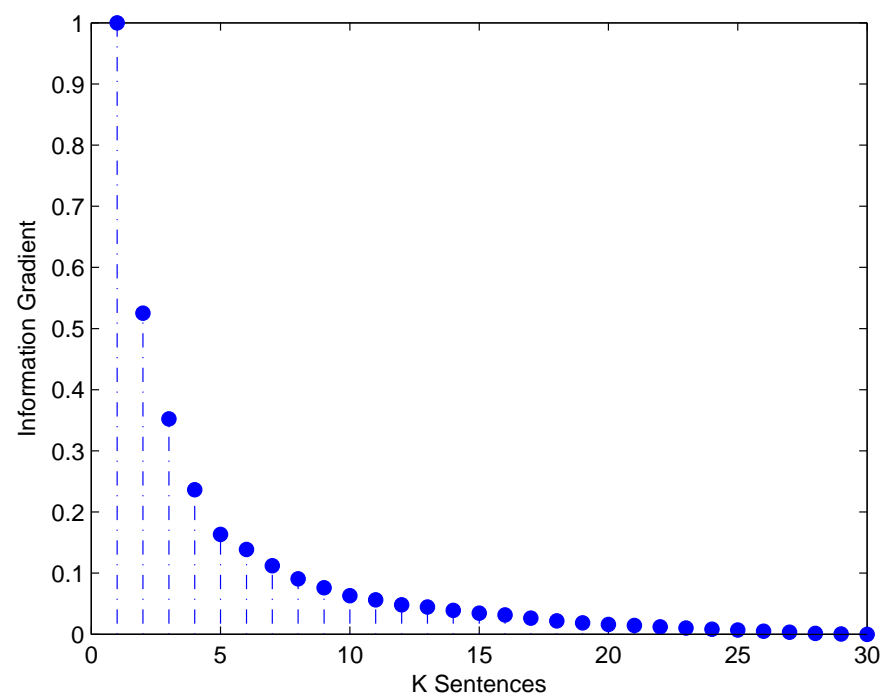

Figure 5: The normalized information gradient of $k^{t h}$ sentence $(k \leq 30)$ 
Fig.4 shows the results chronologically, the 10 sentences are selected from 31939 microblogs in event "The US surveillance program PRISM" from Sina Weibo. These 10 sentences mainly discuss different aspects of the event: $s_{1}$ answers which companies involving the surveillance program: "Google, Facebook, Microsoft, Apple"; $s_{2}$ is about "USA National Security Bureau monitors China network by the Cisco router"; $s_{3}$ answers "Why European countries refuse Huawei and ZTE to participate in communication network construction"; $s_{4}$ is about "Besides Cisco, 3 other surveillance projects are in china"; $s_{5}$ is about "Since British Intelligence Agency monitors foreign official telephone and computer, Russia and Turkey asked British to give explanation"; $s_{6}$ is about "Prism promoted information equipment localization"; $s_{7}$ is about "network security received increasing attention after Prism"; $s_{8}-s_{10}$ mainly about "the whereabouts of Snowden".

Dividing information gradient by the maximum value, we normalizes the information gradient. Fig. 5 shows the normalized information gradient of $k^{t h}$ sentence $(1 \leq k \leq 30)$. It shows that the value of information gradient decreases from 1 to a stable value which is approaching to 0 . As more sentences are selected as core semantics of an event, the incoming sentence contains lower information gradient since most semantic association relations of the event have been described. The most of semantic association is covered by the first 15 sentences and the information gradient of remains sentences is extremely weak.

\subsection{Experimental results}

To evaluate our method on dataset 1 , we compare our method with two baseline methods under three measurements as described in section6.1. We extract $k$ sentences $(1 \leq k \leq 15)$ from each topic by our method and other two baseline methods. Table 4 compares the three methods by ROUGE-1, ROUGE- 2 and ROUGE-SU4 under $k$ sentences. Table 4 shows that our method always outperforms other two baseline methods on ROUGE-1, ROUGE-2 and ROUGE-SU4

\footnotetext{
${ }^{5}$ http://nlp.stanford.edu/software/lex-parse.shtml
} 

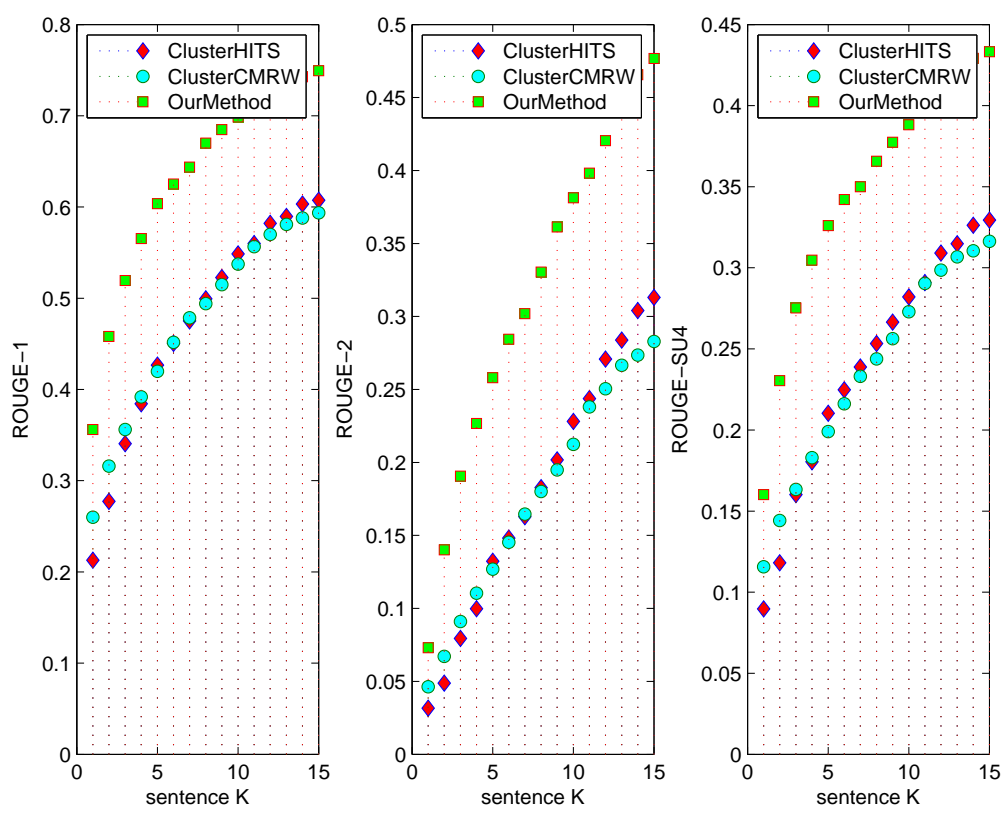

Figure 6: ROUGE-1,ROUGE-2 and ROUGE-SU4 between ClusterHITS, ClusterCMRW and Our method in dataset 1 
Table 4: Comparison of different core semantics discovery methods on dataset 1

\begin{tabular}{|c|c|c|c|c|c|}
\hline \multicolumn{2}{|c|}{ Evaluate measurement } & ClusterCMRW & ClusterHITS & Our method & Sig. \\
\hline \multirow{7}{*}{ Rouge-1 } & 2 & 0.3159 & 0.2773 & 0.4582 & 0.00 \\
\hline & 4 & 0.3918 & 0.3842 & 0.5653 & 0.00 \\
\hline & 6 & 0.4516 & 0.4505 & 0.6252 & 0.00 \\
\hline & 8 & 0.4941 & 0.4994 & 0.6701 & 0.01 \\
\hline & 10 & 0.5373 & 0.5486 & 0.6986 & 0.00 \\
\hline & 12 & 0.5700 & 0.5820 & 0.7218 & 0.00 \\
\hline & 14 & 0.5879 & 0.6033 & 0.7427 & 0.00 \\
\hline \multirow{7}{*}{ Rouge-2 } & 2 & 0.0671 & 0.0489 & 0.1401 & 0.01 \\
\hline & 4 & 0.0910 & 0.0794 & 0.2267 & 0.00 \\
\hline & 6 & 0.1104 & 0.0998 & 0.2844 & 0.00 \\
\hline & 8 & 0.1269 & 0.1323 & 0.3304 & 0.00 \\
\hline & 10 & 0.1453 & 0.1481 & 0.3814 & 0.00 \\
\hline & 12 & 0.1645 & 0.1629 & 0.4205 & 0.00 \\
\hline & 14 & 0.1801 & 0.1828 & 0.4656 & 0.00 \\
\hline \multirow{7}{*}{ Rouge-SU4 } & 2 & 0.1181 & 0.1181 & 0.2304 & 0.01 \\
\hline & 4 & 0.1602 & 0.1602 & 0.2752 & 0.00 \\
\hline & 6 & 0.1804 & 0.1804 & 0.3048 & 0.00 \\
\hline & 8 & 0.2103 & 0.2103 & 0.3261 & 0.00 \\
\hline & 10 & 0.2248 & 0.2248 & 0.3421 & 0.00 \\
\hline & 12 & 0.2388 & 0.2388 & 0.3500 & 0.00 \\
\hline & 14 & 0.2533 & 0.2533 & 0.3658 & 0.00 \\
\hline
\end{tabular}



periods as reference data of dataset 2 since the headlines are condensed for the news. We expect that the core semantics of an event should be contained by these news titles. To verify our method is efficient on dataset 2 , we conduct experiments as follows: For each event in dataset 2, we extract $k$ sentences ${ }_{415}(0<k \leq 30)$ from each event by our method and two other baseline methods respectively. For each event, we use the title of Baidu news of this event as reference data. We calculate gram-1, gram-2 and gram-SU4 by comparing machine generated sentences with title of Baidu news for each event. We compare our method with two baseline methods under ROUGE-1, ROUGE-2 and significantly preforms better than other baseline methods with significant value (sig. $\leq 0.01)$.

Fig.7 shows the comparison of our method with other baseline methods on ROUGE-1,ROUGE-2 and ROUGE-SU4. We compare these methods under $k$ ${ }_{425}$ sentences $(0<k \leq 30)$. It shows that our method obtain higher ROUG-1, ROUG-4 value than other methods do before 23 sentences; after 23 such advantages get week. Such phenomenon is caused by that the core semantics of these events have been coved by the 23 sentences and that adding new sentences dose not increase information gradient. However, our method has higher ROUGE2 than other two methods. From the above analysis, we can verify that our method performs better for discovering the core semantics of event. 
Table 5: Comparison of different core semantics discovery methods on dataset2

\begin{tabular}{|c|c|c|c|c|c|}
\hline \multicolumn{2}{|c|}{ Evaluate measurement } & ClusterCMRW & ClusterHITS & Our method & Sig. \\
\hline \multirow{7}{*}{ Rouge-1 } & 2 & 0.06994 & 0.08817 & 0.15833 & 0.00 \\
\hline & 4 & 0.08987 & 0.14667 & 0.17528 & 0.00 \\
\hline & 6 & 0.13014 & 0.16765 & 0.22743 & 0.00 \\
\hline & 8 & 0.18694 & 0.22742 & 0.22340 & 0.00 \\
\hline & 10 & 0.18991 & 0.23124 & 0.26261 & 0.00 \\
\hline & 12 & 0.24205 & 0.23802 & 0.29207 & 0.00 \\
\hline & 14 & 0.25265 & 0.25159 & 0.30161 & 0.01 \\
\hline \multirow{7}{*}{ Rouge-2 } & 2 & 0.00128 & 0.00180 & 0.00385 & 0.01 \\
\hline & 4 & 0.00333 & 0.003078 & 0.00487 & 0.00 \\
\hline & 6 & 0.00487 & 0.004104 & 0.00590 & 0.00 \\
\hline & 8 & 0.00590 & 0.004360 & 0.00795 & 0.00 \\
\hline & 10 & 0.00641 & 0.00693 & 0.00821 & 0.00 \\
\hline & 12 & 0.00667 & 0.00821 & 0.00923 & 0.00 \\
\hline & 14 & 0.00769 & 0.00821 & 0.01231 & 0.00 \\
\hline \multirow{7}{*}{ Rouge-SU4 } & 2 & 0.03189 & 0.03945 & 0.06954 & 0.01 \\
\hline & 4 & 0.04134 & 0.06548 & 0.07800 & 0.00 \\
\hline & 6 & 0.05927 & 0.07476 & 0.10097 & 0.00 \\
\hline & 8 & 0.08413 & 0.09908 & 0.10142 & 0.00 \\
\hline & 10 & 0.08539 & 0.10404 & 0.11665 & 0.00 \\
\hline & 12 & 0.10809 & 0.10773 & 0.12998 & 0.00 \\
\hline & 14 & 0.11304 & 0.11638 & 0.13502 & 0.00 \\
\hline
\end{tabular}



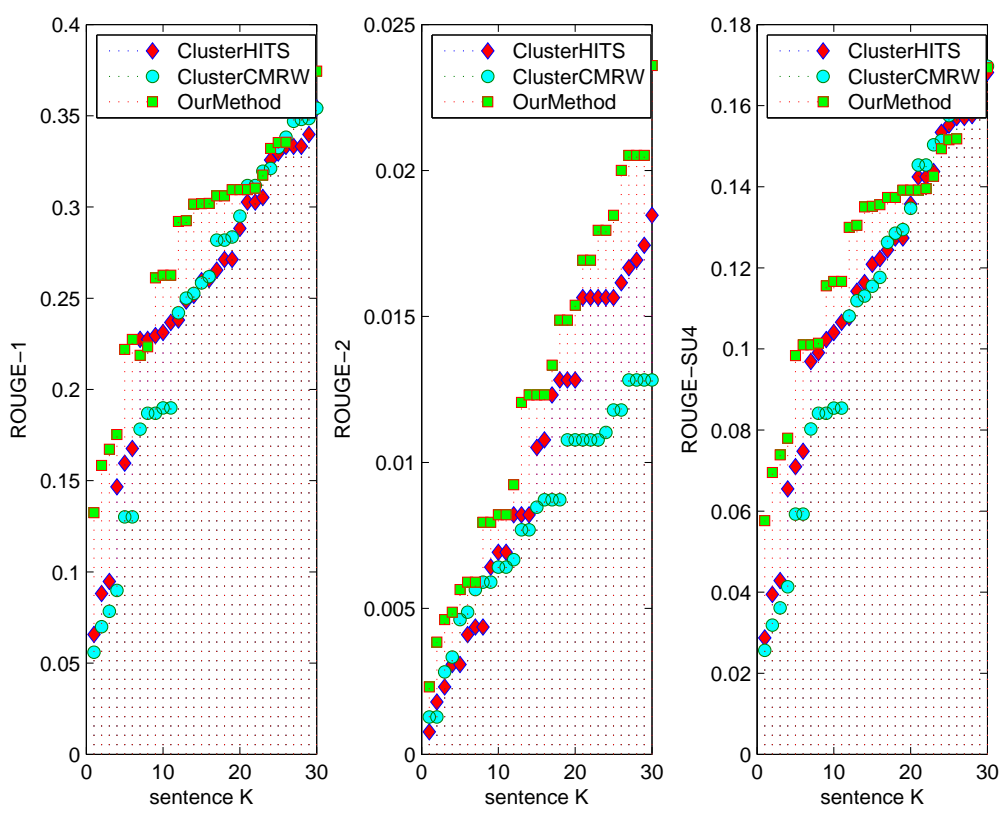

Figure 7: ROUGE-1,ROUGE-2 and ROUGE-SU4 between ClusterHITS, ClusterCMRW and Our method in dataset 2 


\section{Conclusion and future work}

With widely use of open social media such as Twitter and Sina Weibo, the occurrence of a real-world hot event often causes large volumes of user-contributed

435 core semantics of event becomes a challenging problem since the core semantics of an event has been flooded by volumes of short texts which contain redundancy, noise and irrelevant content. The major challenges include:

1) how to learn association relations distribution by small-scale association re440

2) how to maximize coverage of association relation distribution by the minimum number of short texts.

To solve the above challenging issues, the Markov random field based method extracts $k$ sentences as the core semantics of event by,

1) semantic collaborative computation between event Markov random field and event power serial representation, which obtains association distribution by small scale association relations efficiently.

2 ) information gradient computation for maximizing information gradient of $k$ sentences, which generates redundancy-free results by maximizing information gradient with the minimum number of short texts.

To evaluate our method, we compare our method with other state-of-theart methods on TAC standard dataset and a large scale microblog dataset. The results show that our method outperforms other two baseline methods in discovering the core semantics of event.

Some users may consider how to organize these extracted short texts in semantic coherent way and others may focus on what's the influence of other factors for discovering core semantics, such as temporal factor, user information and so on. We plan to explore these problems in future work. 


\section{References}

460

[1] A. Nenkova, K. McKeown, A survey of text summarization techniques, in: Mining Text Data, Springer, 2012, pp. 43-76.

[2] L. Vanderwende, H. Suzuki, C. Brockett, A. Nenkova, Beyond sumbasic: Task-focused summarization with sentence simplification and lexical expansion, Information Processing \& Management 43 (6) (2007) 1606-1618.

[8] V. Qazvinian, D. R. Radev, Scientific paper summarization using citation summary networks, in: Proceedings of the 22nd International Conference on Computational Linguistics-Volume 1, Association for Computational Linguistics, 2008, pp. 689-696. 
[9] D. Shen, J.-T. Sun, H. Li, Q. Yang, Z. Chen, Document summarization using conditional random fields., in: IJCAI, Vol. 7, 2007, pp. 2862-2867.

[10] X. Cai, W. Li, R. Zhang, Combining co-clustering with noise detection for theme-based summarization, ACM Transactions on Speech and Language Processing (TSLP) 10 (4) (2013) 16.

[11] G. Kumaran, J. Allan, Text classification and named entities for new event detection, in: Proceedings of the 27th annual international ACM SIGIR conference on Research and development in information retrieval, ACM, 2004, pp. 297-304.

[12] H. Becker, M. Naaman, L. Gravano, Selecting quality twitter content for events., ICWSM 11.

[13] R. Irfan, C. K. King, D. Grages, S. Ewen, S. U. Khan, S. A. Madani, J. Kolodziej, L. Wang, D. Chen, A. Rayes, et al., A survey on text mining in social networks, The Knowledge Engineering Review 30 (02) (2015) 157170.

[14] Y. Sun, J. Han, J. Gao, Y. Yu, itopicmodel: Information networkintegrated topic modeling, in: Data Mining, 2009. ICDM'09. Ninth IEEE International Conference on, IEEE, 2009, pp. 493-502.

[15] S. Basu, I. Davidson, K. Wagstaff, Constrained clustering: Advances in algorithms, theory, and applications, CRC Press, 2008.

[16] H. Zhuge, Communities and emerging semantics in semantic link network: Discovery and learning, Knowledge and Data Engineering, IEEE Transactions on 21 (6) (2009) 785-799.

[17] X. Luo, J. Zhang, F. Ye, P. Wang, C. Cai, Power series representation model of text knowledge based on human concept learning, Systems, Man, and Cybernetics: Systems, IEEE Transactions on 44 (1) (2014) 86-102. 
[18] X. Luo, Z. Xu, J. Yu, X. Chen, Building association link network for semantic link on web resources, Automation Science and Engineering, IEEE Transactions on 8 (3) (2011) 482-494.

[19] H. Zhuge, Interactive semantics, Artificial Intelligence 174 (2) (2010) 190204.

[20] H. Zhuge, Cyber-physical societythe science and engineering for future society, Future Generation Computer Systems 32 (0) (2014) 180 - 186.

[21] B. Xu, H. Zhuge, Automatic faceted navigation, Future Generation Computer Systems 32 (0) (2014) 187 - 197.

[22] J. Chen, H. Zhuge, Summarization of scientific documents by detecting common facts in citations, Future Generation Computer Systems 32 (0) (2014) $246-252$.

[23] H. Zhuge, Semantic linking through spaces for cyber-physical-socio intelligence: A methodology, Artificial Intelligence 175 (5) (2011) 988-1019.

[24] A. Celikyilmaz, D. Hakkani-Tür, Discovery of topically coherent sentences for extractive summarization, in: Proceedings of the 49th Annual Meeting of the Association for Computational Linguistics: Human Language Technologies-Volume 1, Association for Computational Linguistics, 2011, pp. $491-499$.

530 [25] D. Das, A. F. Martins, A survey on automatic text summarization, Literature Survey for the Language and Statistics II course at CMU 4 (2007) $192-195$.

[26] S. Tabassum, E. Oliveira, A review of recent progress in multi document summarization, in: Doctoral Symposium in Informatics Engineering, 2015.

${ }_{535}[27]$ B. Hayes-Roth, F. Hayes-Roth, Concept learning and the recognition and classification of exemplars, Journal of Verbal Learning and Verbal Behavior 16 (3) (1977) 321-338. 
[28] J. P. Minda, J. D. Smith, Comparing prototype-based and exemplar-based accounts of category learning and attentional allocation., Journal of Experimental Psychology: Learning, Memory, and Cognition 28 (2) (2002) 275.

[29] Y. Yao, Interpreting concept learning in cognitive informatics and granular computing, Systems, Man, and Cybernetics, Part B: Cybernetics, IEEE Transactions on 39 (4) (2009) 855-866.

[30] J. Feldman, The simplicity principle in human concept learning, Current Directions in Psychological Science 12 (6) (2003) 227-232.

[31] J. Feldman, An algebra of human concept learning, Journal of mathematical psychology 50 (4) (2006) 339-368.

[32] J. Feldman, A catalog of boolean concepts, Journal of Mathematical Psychology 47 (1) (2003) 75-89.

[33] J. Feldman, Minimization of boolean complexity in human concept learning, Nature 407 (6804) (2000) 630-633.

[34] F. C. T. Chua, S. Asur, Automatic summarization of events from social media., in: ICWSM, Citeseer, 2013.

[35] L. Shou, Z. Wang, K. Chen, G. Chen, Sumblr: continuous summarization of evolving tweet streams, in: Proceedings of the 36th international ACM SIGIR conference on Research and development in information retrieval, ACM, 2013, pp. 533-542.

[36] J. Leskovec, L. Backstrom, J. Kleinberg, Meme-tracking and the dynamics of the news cycle, in: Proceedings of the 15th ACM SIGKDD international conference on Knowledge discovery and data mining, ACM, 2009, pp. 497506.

[37] S. B. Kaleel, A. Abhari, Cluster-discovery of twitter messages for event detection and trending, Journal of Computational Science 6 (2015) 47-57. 
[38] D. Zhou, L. Chen, Y. He, An unsupervised framework of exploring events on twitter: Filtering, extraction and categorization, in: Twenty-Ninth AAAI Conference on Artificial Intelligence, 2015.

[39] X. Zhou, L. Chen, Event detection over twitter social media streams, The VLDB JournalThe International Journal on Very Large Data Bases 23 (3) (2014) 381-400.

[40] G. Petkos, S. Papadopoulos, Y. Kompatsiaris, Two-level message clustering for topic detection in twitter., in: SNOW-DC@ WWW, 2014,pp. 49-56.

[41] M. I. Jordan, Graphical models, Statistical Science (2004) 140-155.

[42] D. Pavlov, H. Mannila, P. Smyth, Beyond independence: Probabilistic models for query approximation on binary transaction data, Knowledge and Data Engineering, IEEE Transactions on 15 (6) (2003) 1409-1421.

[43] F. Jelinek, Statistical methods for speech recognition, MIT press, 1997.

[44] J. N. Darroch, D. Ratcliff, Generalized iterative scaling for log-linear models, The annals of mathematical statistics (1972) 1470-1480.

[45] D. Pavlov, H. Mannila, P. Smyth, Probabilistic models for query approximation with large sparse binary data sets, in: Proceedings of the Sixteenth conference on Uncertainty in artificial intelligence, Morgan Kaufmann Publishers Inc., 2000, pp. 465-472.

[46] C. Huang, A. Darwiche, Inference in belief networks: A procedural guide, 585

[47] D. Rundus, Analysis of rehearsal processes in free recall., Journal of experimental psychology 89 (1) (1971) 63.

[48] J. Leskovec, A. Krause, C. Guestrin, C. Faloutsos, J. VanBriesen, N. Glance, Cost-effective outbreak detection in networks, in: Proceedings of the 13th ACM SIGKDD international conference on Knowledge discovery and data mining, ACM, 2007, pp. 420-429. 
[49] C.-Y. Lin, E. Hovy, Automatic evaluation of summaries using n-gram cooccurrence statistics, in: Proceedings of the 2003 Conference of the North American Chapter of the Association for Computational Linguistics on 595 Human Language Technology-Volume 1, Association for Computational Linguistics, 2003, pp. 71-78. 